Isidore Murhi Mihigo

Université catholique de Bukavu, RDC

Célestin Bucekuderhwa Bashige

Université catholique de Bukavu, RDC

\section{Abandon scolaire au Sud-Kivu}

doi:10318162/fp.2017.418

\section{R ésumé}

L'abandon scolaire fait l'objet de plusieurs études à l'échelle internationale et intéresse plusieurs disciplines. L'objectif du présent article est de dégager les éléments expliquant l'abandon précoce de l'école au Sud-Kivu. Les données utilisées dans cet article viennent de la division provinciale de l'enseignement primaire, secondaire et professionnel (EPSP), d'une part, et de l'enquête 1-2-3 de 2005, d'autre part. Nous appuyant sur une analyse économétrique multiniveau, les résultats montrent que plus on commence l'école avec du retard, plus on risque de décrocher. Les caractéristiques associées à l'élève et à la famille sont aussi prépondérantes dans l'abandon des élèves au Sud-Kivu.

Mots-clés

Abandon scolaire, décrochage scolaire, Sud-Kivu, primaire, secondaire, éducation

Abstract

School dropout has been tackled in many research studies internationally, and from several disciplinary perspectives. This paper attempts to identify the factors pushing students to leave school prematurely in South-Kivu Province. To this end, we analyzed data from the archives of the Provincial division of Education (known by its French acronym EPSP) and the 2005 1-2-3 survey data, drawing on the multilevel econometric approach. Results show that the later a child starts school, the higher the probability that he or she drops out. Characteristics associated with the student, family and school play a predominant role in school dropout in South-Kivu.

\section{Keywords}

School dropout, South-Kivu, primary, secondary, education

\section{Introduction}

L'accès à l'éducation préoccupe actuellement l'humanité et l'importance de l'éducation a été prouvée à maintes reprises. La Conférence mondiale sur l'Éducation pour tous (Jomtien, en 1990) avait reconnu comme priorité des priorités l'accès à l'éducation et l'amélioration de la qualité de l'éducation des enfants, des jeunes et des adultes (Ananga, 2011; UNESCO, 2009). C'est dans l'après-Seconde Guerre mondiale (Isambert-Jamati, 1992) que l'obtention d'un diplôme devint nécessaire pour participer à la vie sociale et espérer un futur meilleur. Cependant, malgréles efforts considérables déployés pour garantirl'accès àl'éducation, la réalité est telle que plusieurs enfants n'ont toujours pas accès à ce droit. Et pour ceux qui y accèdent, nombre d'entre eux n'atteignent pas la fin du cycle débuté. Benny et Frappier (1997) montrent que plusieurs enfants dans le monde n'achèvent pas l'éducation de base qu'ils ont entamée. En Afrique, nombre d'élèves abandonnent l'école avant de finir le cycle primaire (Ananga, 2011; Lloyd, Mensch et Clark, 2000; UNESCO, 2010, 2012). Les problèmes éducatifs et la manière d'y répondre varient selon les objectifs des pays (Belzil, 2004; CoulidiatiKiélem, 2009; Fry, 2003; Huot et Castonguay, 2014; Muskens, 2009; UNICEF, 2011). Bien que le taux d'accès à l'éducation dans les pays en développement ait augmenté, on est loin d'atteindre l'objectif fixé (Easterly, 2008; Sabates, Hossain et Lewin, 2010; UNESCO, 2012; UNICEF, 2011). Il apparaît dès lors évident que le niveau d'éducation atteint par un individu dans une société constitue un déterminant majeur de son succès futur (Huot et Castonguay, 2014; Joanis, 2002). Aussi, les Objectifs du Millénaire pour le développement considéraientils l'éducation, la santé et un environnement assaini comme ingrédients nécessaires à une vie agréable et longue (Easterly, 2008; Elliot et Voss, 1974). Le déficit dans l'un ou l'autre de leurs attributs constitue un signe de pauvreté plus grave que la pauvreté monétaire. Cette vision est relayée par les objectifs du développement durable (ODD) aujourd'hui. 
Mais, assurer l'éducation pour tous ne consiste pas seulement à garantir que tous les enfants soient inscrits à l'école (Ananga, 2011). Encore faut-il qu'ils continuent à aller à l'école jusqu'à l'obtention du diplôme ou du certificat sanctionnant le cursus. Dès lors, la question de l'abandon scolaire devient vive, un problème social complexe et qui affecte tout le monde. Par rapport à cette situation, nous voulons comprendre, dans le cadre du présent article, les déterminants de l'abandon scolaire au Sud-Kivu.

La pauvreté et l'absence de financement pour l'éducation constitueraient les plus grands obstacles à l'éducation en République démocratique du Congo en général et au Sud-Kivu particulièrement. Aussi existerait-il des barrières sociales et culturelles qui renforcent le phénomène de décrochage scolaire. Si, en Occident, l'abandon scolaire préoccupe et est combattu par le pouvoir public (Larivée et Garnier, 2014), en Afrique, la question de l'abandon scolaire demeure une réalité courante, sans politique appropriée et elle aurait des répercussions sur le devenir du continent. Marcotte (2013) relève que la question d'abandon scolaire a des conséquences sociales inimaginables.

Une abondante littérature sur l'abandon scolaire existe (Ananga, 2011; Belzil, 2004; Bouchard, 2001; Coulidiati-Kiélem, 2009; Gueddari, 2015; Issidor, 2006; Marcotte, 2013; Musisi, Kasente et Balihuta, 2008; Vitaro, Larocque, Janosz et Tremblay, 2001). Issidor (2006) montre que les travaux consacrés à l'économie de l'éducation s'intéressent aussi à l'abandon précoce de l'école qui est un véritable fléau pour la société. Coulidiati-Kiélem (2009) estime que l'abandon scolaire fait subir aux individus et à la société entière d'importants coûts. L'abandon précoce de l'école contribuerait à l'analphabétisme répandu (Benny et Frappier, 1997), au chômage voire au crime dans certaines sociétés. Aux ÉtatsUnis et au Burkina Faso par exemple, des études (Coulidiati-Kiélem, 2009; Dei, Mazzuca, Mclsaac et Zine, 1997; Sum, Khatiwada, McLaughlin et Palma, 2009) montrent que les garçons quittent plus prématurément l'école que les filles. En République démocratique du Congo et au Sud-Kivu particulièrement, Ntagoma et Lukuli (2008) affirment que la question éducative doit préoccuper tout le monde, car la dégradation de l'environnement éducatif conduit au sous-développement. Murhi Mihigo (2016) indique que la prise en charge financière des enseignants par les parents au Congo a des conséquences néfastes et prive beaucoup d'enfants de scolarisation. La problématique de l'abandon scolaire au Congo est moins documentée alors que ses effets néfastes sont légion.

L'objectif du présent article est de faire un état des lieux de l'abandon scolaire au Sud-Kivu et de relever les raisons qui poussent les élèves à abandonner précocement l'école.

L'approche méthodologique adoptée dans cette étude est inspirée de Belzil (2004), Boutin (2010), Coulidiati-Kiélem (2009), Courgeau (2004), Durand (2006) et Rumberger (1995), qui examinent le phénomène à l'aide de modèles d'analyse multiniveau ${ }^{2}$.

Cet article comprend quatre sections. La première section présente une revue de la littérature, la deuxième est consacrée au contexte de la province du Sud-Kivu de 2005 à 2009 et la troisième aborde la méthodologie. Dans la dernière section, nous interprétons les résultats. 


\section{Revue de littérature}

L'abandon scolaire n'est pas perçu de la même manière par les auteurs (Chávez, Belkin, Hornback et Adams, 1991). Le concept connait une diversité de définitions selon les auteurs et le contexte. Boissonneault, Michaud, Côté, Tremblay et Allaire (2007) perçoivent l'abandon scolaire comme un manque de réussite ou simplement de l'insuccès. En Slovaquie, l'abandon scolaire concerne les élèves qui nont pas terminé l'enseignement de base. Au Liban, l'abandon scolaire est associé à un élève qui quitte le cursus avant son terme, pour toute autre raison que la mort, sans être inscrit dans une autre école (Ananga, 2011). Au Ghana, Fentiman (1999), cité dans Ananga (2011), définit un décrocheur comme un élève qui n'a pas terminé les neuf ans d'éducation de base. Le décrochage se rapproche ainsi de la "déscolarisation " (Boissonneault et al., 2007). De nombreux termes sont utilisés par les chercheurs. On parle par exemple chez les anglophones de la « educational death " (Chávez et al., 1991). On fait référence aussi à la « démobilisation scolaire » (Bouchard, 2001; Musisi et al., 2008), à

la « désaffiliation » ou à la « non-affiliation »(Chávez et al., 1991; Lee et Burkam, 2000). Est considéré comme "décrocheur " tout élève qui était inscrit au début d'une année scolaire et qui ne l'est plus l'année suivante sans être titulaire d'un diplôme d'études secondaires. Les décès et les déménagements à l'étranger sont exclus de la catégorie (Boissonneault et al., 2007). Un élève est dit « à risque d'abandon scolaire " lorsqu'il fréquente toujours l'école, mais présente une forte probabilité de décrochage. Au Congo, tout élève qui commence les études et qui arrête avant d'obtenir le diplôme ou le certificat qui sanctionne la fin du cycle est qualifié de décrocheur. Dagenais, Montmarquette, Viennot-Briot et Meunier (2010) proposent de retenir le nombre de trois semaines d'absences continues non motivées pour identifier un décrocheur. Cela conduit à distinguer les types de décrocheurs.

Sawadogo et Soura (2002) font une distinction entre les décrocheurs handicapés sur le plan intellectuel (ayant un retard intellectuel ou une déficience intellectuelle), qu'on qualifie de décrocheurs involontaires ${ }^{3}$ et les décrocheurs ayant la capacité intellectuelle, mais qui ont abandonné l'école pour de raisons indépendantes de leur volonté, mais liées à l'environnement socioéconomique (maladie, mortalité, manque de moyen financier...). Duchesne et Thomas (2005) proposent six types de décrocheurs :

- les « drop-outs accidentels », qui ont toutes les capacités voulues pour terminer leurs études, mais préfèrent aller sur le marché du travail et obtenir une rémunération au lieu de poursuivre des études abstraites;

- $\quad$ les inadaptés, qui éprouvent trop de difficultés intellectuelles, motrices ou comportementales pour s'adapter à l'école;

- les défavorisés, qui évoluent dans un milieu socioéconomique et familial défavorisé assombrissant tellement les perspectives d'avenir que l'école en perd son sens;

- $\quad$ les délinquants, qui ressemblent aux défavorisés, mais qui ont développé en plus des conduites sociales inadaptées;

- les «drop-outs féminins », qui abandonnent l'école à cause du mariage ou des grossesses précoces;

- les marginaux, des adolescents qui ont tout pour eux (intelligence, aisance familiale, habiletés créatrices, etc.), mais qui n’arrivent pas à s'épanouir à l'école. 
Duchesne et Thomas (2005) distinguent aussi les décrocheurs pour cause d'emploi et ceux qui accumulent les échecs scolaires. Elliot et Voss (1974) parlent des délinquants scolaires. Gueddari (2015) affirme aussi que l'abandon scolaire peut être dû au milieu défavorisé.

Les causes de ce phénomène sont multidimensionnelles. Les psychologues mettent l'accent sur les dimensions intrapsychiques, comportementales et socio-interactionnelles. Les économistes s'intéressent aux éléments liés à la pédagogie, à l’environnement éducatif et socioéconomique.

Murhi Mihigo (2016) montre que l'intervention limitée de l'État dans le secteur éducatif est à la base de plusieurs abandons scolaires au Congo. Ainsi, le phénomène d'abandon prématuré de l'école conduit à des conséquences qui affectent non seulement la personne concernée, mais toute la société.

Les analyses actuelles de l'abandon scolaire sont appréhendées avec l'approche multiniveau. La mise en œuvre de l'analyse multiniveau permet de savoir si les relations entre les individus et les contextes sont de nature causale (Rumberger, 1995). Dans le même ordre d'idées, Bressoux, Coustère et LeroyAudouin (1997) affirment que l'un des domaines de prédilection pour l'analyse multiniveau est le domaine de l'éducation.

Pour les sociologues, l'intérêt est du côté de la construction sociale du phénomène, le rôle des politiques, les facteurs structurant les institutions et les rapports entre classes (Bushnik, Barr-Telford et Bussière, 2004; Lloyd et al., 2000). D'autres chercheurs indiquent que le décrochage scolaire découle de facteurs multiples : individuels, scolaires (Forget et Lehraus, 2015; Jeffrey,2013), familiaux ou environnementaux (Belzil, 2004; Bushnik et al., 2004; Dei et al., 1997; Gueddari, 2015; Jimerson, Egeland, Sroufe et Carlson, 2000; Rosenthal, 1998). L'analyse par des modèles classiques (mononiveau) de différents facteurs n'est plus appropriée, voilà pourquoi il faut introduire l'analyse multiniveau qui facilite le traitement simultané des données appartenant à plusieurs niveaux hiérarchisés (Bressoux et al., 1997).

Les conséquences de l'abandon scolaire varient considérablement en fonction des individus, des sociétés et des milieux de vie (Gueddari, 2015). Nombre d'études (Lee et Burkam, 2000; Sum et al., 2009) démontrent que les élèves interrompant leurs études précocement courent de risques d'éprouver des problèmes sociaux, économiques et sanitaires. L'intégration socioprofessionnelle de ceux qui ont abandonné les études est plus difficile. Ils sont plus nombreux à réclamer de l'aide sociale et leurs emplois sont moins stables, moins prestigieux et moins rémunérés (Sum et al., 2009). Les décrocheurs devenant parents, ayant été moins scolarisés eux-mêmes, courent de plus grands risques que leur progéniture éprouve à son tour des difficultés scolaires (Marcotte, 2013; Sabates, Hossain et al., 2010). Le décrochage scolaire conduit ainsi à une crise intergénérationnelle. L'abandon scolaire est aussi contextuel, car chaque milieu l'appréhende de sa manière (Gueddari, 2015). Le modèle multiniveau propose une solution adéquate au problème longtemps posé dans le domaine de l'éducation, celui de rendre compte du fait que l'individu s'insère dans un milieu qui ne peut manquer d'influence sur lui.

Il est désormais possible d'articuler dans un même modèle d'analyse différents niveaux d'observation. Contrairement aux modèles contextuels, où toutes les caractéristiques sont analysées au niveau individuel, Courgeau (2004) trouve que le modèle multiniveau fait référence à une structuration des données, et donc du comportement analysé selon différents niveaux d'observation et d'analyse. L'analyse multiniveau constitue donc un pas de géant dans la modélisation de la réalité sociale de l'ère. L'approche multiniveau permet de mesurer l'importance relative de différents niveaux, 
et d'identifier les niveaux pertinents pour l'analyse (Bressoux et al., 1997; Courgeau, 2004). Le modèle multiniveau ne nécessite pas de base de données très lourde, et permet aussi d'envisager la collecte sur de petits effectifs. Un autre avantage réside dans l'analyse conjointe de données d'origines différentes, comme c'est le cas dans cette étude. Le développement du modèle est fait dans la section sur l'approche méthodologique.

\section{Contexte de la scolarité dans la province du Sud-Kivu}

Au Congo, l'offre de l'éducation, assurée autrefois par l'État congolais, est assurée actuellement conjointement avec d'autres partenaires, dont les organisations religieuses et les particuliers (Murhi Mihigo, 2016) ${ }^{4}$. Le système actuel conduit vers la multiplication de structures d'enseignement, ce qui augmente les possibilités d'offres scolaires. En dépit de l'augmentation d'établissements, la division provinciale de l'enseignement primaire, secondaire et professionnel (tableaux 1 et 3) constate un déficit de l'offre par rapport à la demande en le liant au taux de scolarisation. Le taux de scolarisation est le pourcentage obtenu à l'aide du rapport entre les effectifs d'élèves et la population scolarisable correspondante d'un cycle donné (Diagne, Kafando et Ounteni, 2006; Durand, 2006).

Nous analysons le taux d'admission ${ }^{5}$. Au Sud-Kivu, le taux d'admission en première année d'enseignement primaire a augmenté depuis 2005 et avoisine les $80 \%$ en 2009. Il en est de même pour les filles que pour les garçons. Ceci peut sembler permettre d'affirmer que la population scolarisable est scolarisée à près de $80 \%$. Ces chiffres doivent être nuancés lorsqu'on sait que les inscrits en première ne sont pas seulement ceux ayant l'âge d'admission légale de 6 ans (Murhi Mihigo, 2016). En première année d'enseignement primaire, il y a aussi des élèves de moins de 6 ans et des élèves ayant plus de 7 ans. L'UNICEF (2011) montre que, sur les inscrits en première, $40 \%$ ont l'âge de 6 ans (âge légal), tandis que les enfants âgés de plus de 6 ans sont près de $60 \%$ d'inscrits. Ntagoma et Lukuli (2008) trouvent aussi que la majorité des enfants sont inscrits à l'école avec du retard par rapport à l'âge légal. Les contraintes financières sont la base d'un faible niveau de scolarisation. Pour Huot et Castonguay (2014), un milieu défavorisé peut être une cause de non-scolarisation chez les enfants. En Afrique subsaharienne, près de 30 millions d'enfants en âge d'être scolarisés sont non scolarisés (UNESCO, 2012).

La différence de taux entre garçons et filles varie selon les milieux. Selon une étude réalisée au Nigeria, les populations rurales limitent la scolarisation de leurs filles, car celle-ci est perçue comme une menace pour leur identité (Durand, 2006). Avec l'accélération de la campagne «Éducation pour tous » et celle de l'UNICEF « toutes les filles à l'école » de 2006, le taux d'admission a augmenté. Le taux d'admission en première année d'enseignement primaire a plus augmenté pour les filles (de $50 \%$ en 2005 à $70 \%$ en 2009) que pour les garçons (de $83 \%$ en 2005 à $91 \%$ en 2009). Les mesures visant à éliminer les obstacles économiques à l'éducation sont intégrées dans les plans d'action annuels du Document de la stratégie de croissance et de réduction de la pauvreté (DSCRP) (UNICEF, 2011). 


\section{Tableau 1}

Scolarisation de la tranche de 7 à 12 ans (école primaire).

\section{Nombre d'élèves}

Estimation des enfants de 7 à 12 ans

\begin{tabular}{llllllllll}
\hline Année & Garçons & Filles & Total & Garçons & Filles & Total & Garçons & Filles & Total \\
\hline $\mathbf{2 0 0 5}$ & 316223 & 291419 & 607642 & 484000 & 503000 & 987000 & 65 & 58 & 62 \\
\hline $\mathbf{2 0 0 6}$ & 394801 & 325678 & 720479 & 525000 & 562800 & 1087872 & 75 & 58 & 66 \\
\hline $\mathbf{2 0 0 7}$ & 395200 & 331782 & 726982 & 412000 & 410000 & 822000 & 96 & 81 & 88 \\
\hline $\mathbf{2 0 0 8}$ & 428412 & 380021 & 808433 & 528000 & 425000 & 853000 & 81 & 89 & 95 \\
\hline $\mathbf{2 0 0 9}$ & 420868 & 370590 & 791458 & 440185 & 439674 & 879860 & 96 & 84 & 90
\end{tabular}

Source : Annuaires de la Division provinciale de l'EPSP 2005-2009.

Le taux de scolarisation a connu une croissance sur les cinq ans. Chez les garçons, les effectifs scolarisés sont passés de 316223 en 2005 à 420868 élèves en 2009, et le taux de scolarisation a augmenté de plus de $30 \%$. Chez les filles, le nombre d'élèves est passé de 291419 en 2005 à 370590 en 2009, et le taux de scolarisation a augmenté de moins de $30 \%$. Ntagoma et Lukuli (2008) avancent certains facteurs influençant négativement la scolarisation : les frais scolaires que doivent payer les parents et les coutumes constituent des obstacles à la scolarisation des enfants dans un pays où le produit intérieur brut par habitant est faible. Nous avons montré (Murhi Mihigo, 2016) que le fait que l'accès à l'éducation au Congo soit conditionné par la prise en charge financière des enseignants par les parents prive de nombreuses familles pauvres de scolarisation pour leurs enfants.

\section{Tableau 2}

Scolarisation de la tranche de 13-18 ans (secondaire).

\begin{tabular}{cccccccccc}
\multicolumn{2}{l}{ Nombre d'élèves } & \multicolumn{3}{c}{ Estimation des enfants de $\mathbf{1 3}$ à $\mathbf{1 8}$ ans } & \multicolumn{3}{c}{ Taux de scolarisation en \% } \\
\hline Année & Garçons & Filles & Total & Garçons & Filles & Total & Garçons & Filles & Total \\
\hline $\mathbf{2 0 0 5}$ & 158963 & 74126 & 233089 & 412000 & 314000 & 726000 & 39 & 24 & 32 \\
\hline $\mathbf{2 0 0 6}$ & 175022 & 89130 & 264152 & 425000 & 211000 & 636000 & 41 & 42 & 42 \\
\hline $\mathbf{2 0 0 7}$ & 124336 & 64611 & 188947 & 303000 & 252000 & 555000 & 41 & 26 & 34 \\
\hline $\mathbf{2 0 0 8}$ & 146763 & 81224 & 227987 & 288000 & 282000 & 570000 & 51 & 29 & 40 \\
\hline $\mathbf{2 0 0 9}$ & 160326 & 90737 & 251063 & 328900 & 325500 & 654400 & 49 & 28 & 38
\end{tabular}

Source : Annuaires de la Division provinciale de l'EPSP 2005-2009.

Le taux de scolarisation dans l'enseignement secondaire est inférieur à celui de l'enseignement primaire, que ce soit pour les garçons ou pour les filles. Pour les garçons, le taux de scolarisation a augmenté de près de $10 \%$ entre 2005 et 2009 alors que pour les filles, cette augmentation n'est que de $4 \%$. L'UNICEF (2011) montre que l'amélioration des conditions de vie à travers les organisations non gouvernementales est l'un des éléments explicatifs de la hausse du taux de scolarisation des adolescents. Ce qui est important à signaler aujourd'hui, c'est la tendance soutenue à réduire l'écart aussi bien entre garçons et filles qu'entre filles scolarisées en milieu rural' et en ville (Musisi et al., 2008). Mais malgré l'adoption de plusieurs textes législatifs au Congo, la situation de la femme reste caractérisée par une discrimination dans certaines coutumes. 


\section{Taux d'abandon}

Le taux d'abandon scolaire est l'ensemble d'élèves ayant décroché avant la fin de l'année plus ceux ne revenant pas en début de l'année suivante, qu'ils aient réussi ou non (Sabates, Akyeampong, Westbrook et Hunt, 2010; Vitaro et al., 2001).

Les statistiques scolaires sur l'abandon au Sud-Kivu ne donnent pas tous les détails nécessaires ${ }^{8}$. L'abandon scolaire est une notion qui tient du résidu (Issidor, 2006) et qui se calcule par simple soustraction.

\section{Tableau 3}

Évolution du nombre de décrocheurs scolaires à l'école primaire.

\begin{tabular}{cccccccccc}
\multicolumn{1}{l}{ Effectif d'élèves } & \multicolumn{3}{c}{ Nombre de décrocheurs } & \multicolumn{3}{c}{ Taux de décrochage en $\%$} \\
\hline Année & Garçons & Filles & Total & Garçons & Filles & Total & Garçons & Filles & Total \\
\hline $\mathbf{2 0 0 5}$ & 316223 & 291419 & 607642 & 38014 & 29816 & 67830 & 12 & 10 & 11 \\
\hline $\mathbf{2 0 0 6}$ & 394801 & 325678 & 720479 & 35319 & 31033 & 66352 & 9 & 10 & 9 \\
\hline $\mathbf{2 0 0 7}$ & 395200 & 331782 & 726982 & 46941 & 30021 & 76962 & 12 & 9 & 11 \\
\hline $\mathbf{2 0 0 8}$ & 428412 & 380021 & 808433 & 53772 & 28156 & 81928 & 13 & 7 & 10 \\
\hline $\mathbf{2 0 0 9}$ & 420868 & 370590 & 791458 & 51025 & 36484 & 87509 & 12 & 10 & 11
\end{tabular}

Source : Nos calculs, sur base des données des annuaires de la Division provinciale de l'EPSP 2005-2009.

Nous remarquons que le taux de décrochage à l'école primaire oscille entre 9 et $11 \%$. En examinant de très près le comportement différentiel de l'abandon scolaire, il y a lieu de constater que l'un des handicaps à surmonter chez les filles est l'accès à l'école ${ }^{9}$. L'éducation des filles aurait d'importants bénéfices sociaux, en termes de réduction de la mortalité infantile, de la fertilité des femmes et de la santé-nutrition des enfants (Diagne et al., 2006; Fry, 2003). L'écart entre les sexes en faveur des femmes est aussi une réalité au Lesotho et en Sierra Leone (UNESCO, 2012). En Afrique subsaharienne près de 1/5 d'enfants ayant commencé l'école en 2009 ont abandonné l'école primaire avant de finir un cycle.

\section{Tableau 4}

Évolution du nombre de décrocheurs scolaires à l'école secondaire.

\section{Effectif d'élèves}

\begin{tabular}{cccccccccc}
\hline Année & Garçons & Filles & Total & Garçons & Filles & Total & Garçons & Filles & Total \\
\hline $\mathbf{2 0 0 5}$ & 158963 & 74126 & 233089 & 23017 & 13806 & 36823 & 14 & 19 & 16 \\
\hline $\mathbf{2 0 0 6}$ & 175022 & 89130 & 264152 & 17396 & 17297 & 34693 & 10 & 19 & 13 \\
\hline $\mathbf{2 0 0 7}$ & 124336 & 64611 & 188947 & 19677 & 6635 & 26312 & 16 & 10 & 14 \\
\hline $\mathbf{2 0 0 8}$ & 146763 & 81224 & 227987 & 20399 & 9843 & 30242 & 14 & 12 & 13 \\
\hline $\mathbf{2 0 0 9}$ & 160326 & 90737 & 251063 & 12721 & 11014 & 23735 & 8 & 12 & 9
\end{tabular}

Source : Nos calculs, sur base des données de la Division provinciale de l'EPSP 2005-2009.

Nombre de décrocheurs

Taux de décrochage en $\%$ 
Au niveau de l'école secondaire, les taux d'abandon sont plus élevés qu'à l'école primaire. Les effectifs à l'école secondaire sont très faibles. " [...] en dernière année du premier cycle de l'enseignement secondaire, seulement 13 enfants sur 100 ayant débuté l'école en première année du primaire sont toujours à l'école »(UNESCO, 2012). Pour ce qui est des filles, le taux d'abandon évolue négativement; passant de $19 \%$ en 2005 à $10 \%$ en 2007 avant de remonter à $12 \%$ entre 2008 et 2009. Jeffrey (2013) propose pour limiter le décrochage scolaire que les enseignants veillent à ce que les rites scolaires soient suivis avec attention par tous les élèves.

\section{Approche méthodologique}

En plus des données de l'EPSP détaillées dans la section précédente, pour trouver les déterminants de l'abandon scolaire, cet article utilise la base des données de l'enquête 1-2-3 menée par l'Institut national de la statistique de la République démocratique du Congo entre 2004 et 2005. L'enquête 1-2-3 se base sur une méthodologie développée au début des années 90 à DIAL (Développement, institutions et analyses de long terme). Elle est constituée d'un dispositif de trois enquêtes, touchant des populations statistiques différentes : individus, unités de production et ménages. La première phase de cette enquête est une enquête sur l'emploi, le chômage et les conditions d'activités des ménages (phase 1 : enquête emploi). La deuxième phase consiste à réaliser une enquête spécifique auprès des chefs des unités de production informelles sur leurs conditions d'activité, leurs performances économiques, le mode d'insertion dans le tissu productif et leurs perspectives (phase 2 : enquête sur le secteur informel). La troisième phase est une enquête sur la consommation des ménages. Elle vise à estimer le niveau de vie des ménages, à mesurer le poids des secteurs formel et informel dans leurs consommations, et à analyser les déterminants du choix des différents lieux d'achat (phase 3 : enquête sur la consommation, les lieux d'achat et la pauvreté). Pour une cohérence avec les données de la division de l'EPSP, nous avons isolé la province du Sud-Kivu pour retenir un échantillon de 811 observations seulement après nettoyage. Les informations issues de la base des données de l'EPSP n'ont été retenues que pour l'année 2005. Nous n'avons retenu que les variables qui étaient simultanément associées dans les deux bases de données. Certaines variables ont subi un retraitement pour répondre aux exigences de l'analyse. Les résultats sont obtenus grâce à l'utilisation du logiciel Stata 12.0.

\section{Présentation du modèle}

Léducation constitue, selon la théorie du capital humain, la clé du positionnement dans la vie socioprofessionnelle. Les caractéristiques individuelles, familiales et environnementales sont susceptibles d'aider à limiter et/ou d'influencer un élève dans le processus de décrochage ou de nondécrochage scolaire (Gueddari, 2015; Marcotte, 2013; Sabates, Hossain et al., 2010). Pour évaluer les facteurs à la base du décrochage scolaire, la plupart des auteurs (Belzil, 2004; Coulidiati-Kiélem, 2009; Rumberger, 1995) s'appuient sur un modèle économétrique d'analyse multiniveau.

Afin d'identifier l'effet des variables explicatives sur la probabilité de décrochage scolaire, les variables sont catégorisées en trois niveaux : le niveau de l'élève (1), celui de sa famille (2) et celui de l'école (3). Un modèle Probit univarié est estimé séparément pour chacun de trois niveaux. À la fin, un modèle 
complet permet de comparer les influences de chaque niveau dans la probabilité totale. Le modèle implicite se présente comme suit (Boutin, 2010; Rumberger, 1995) ${ }^{10}$ :

$$
y_{i j}^{i}=\beta_{0}+\sum_{j=1}^{k} \beta_{j} x_{i j}+\varepsilon_{i}
$$

$y_{i}^{i}$ désigne la variable latente non observée représentant la probabilité d'abandonner l'école ou non. Il est considéré $y_{\mathrm{i}}^{i}$ comme une variable binaire, égale à 1 si $y_{\mathrm{ij}}^{i}>0$ et 0 sinon. Les $\varepsilon_{\mathrm{i}}$ représentent les termes d'erreurs, normalement distribués. La fonction cumulative normale de la distribution est donnée :

$$
\begin{gathered}
p_{i}=\operatorname{Pr}\left(y_{i j}=1\right)=\operatorname{Pr}\left[\varepsilon_{i}<-\left(\beta_{0}+\sum_{j=1}^{k} \beta_{j} X_{i j}\right)\right] \\
D^{\prime} \text { où } p_{i}=1-F-\left(\beta_{0}+\sum_{j=1} \beta_{j} X_{i j}^{\prime}\right)
\end{gathered}
$$

Où $\mathrm{P}_{\mathrm{i}}$ désigne la probabilité de décrochage scolaire.

L'hypothèse de normalité des termes d'erreurs (Bourbonnais, 2009), nous permet d'affirmer que 1-F($\mathrm{X})=\mathrm{F}(\mathrm{X})$, la distribution des termes d'erreurs est symétrique. La fonction cumulative de la distribution est :

$$
p_{i}=F\left(\beta_{0}+\sum_{j=1}^{k} \beta_{j} X_{i j}\right)
$$

Chaque $Y_{i}$ résulte d'un processus binomial de probabilité $P_{i}$. Les variables explicatives retenues concernent les caractéristiques liées à l'élève, à sa famille et les caractéristiques scolaires ${ }^{11}$. Les variables relatives à l'élève sont le sexe, l'âge de l'élève, l'échec scolaire et l'âge de début de scolarisation. Dans plusieurs pays africains (Coulidiati-Kiélem, 2009; Lloyd, Mensch et Clark, 2000), la discrimination fille-garçon est une tradition lors de la fréquentation scolaire. Le fait qu'un élève soit plus âgé que les autres dans une classe peut le conduire au décrochage scolaire (Dagenais et al., 2010; Sabates, Hossain et al., 2010). Les déterminants familiaux sont : la taille du ménage, le milieu de résidence, les dépenses allouées à l'éducation, la situation économique de parents, l'éducation des parents, l'âge du chef de ménage et la langue maternelle. Pour les déterminants scolaires, nous avons : la conduite de l'enseignant, l'absentéisme de l'enseignant, les activités parascolaires, le traitement des enseignants, la qualification de l'enseignant et la réputation de l'école, mesurée par le régime de gestion (Forget et Lehraus, 2015). En effet, les écoles conventionnées ont une bonne réputation par rapport aux écoles publiques et à certaines écoles privées (Murhi Mihigo, 2016). Les recherches ont prouvé que le nombre de personnes dans un ménage, la résidence du ménage, le revenu du ménage, les réalités de chaque enseignant sont importants dans l'analyse du décrochage scolaire (Isambart-Jamati, 1992; Larivée et Garnier, 2014; Okumu, Nakajjo et Isoke, 2008; Sum et al., 2009). L'apport du modèle multiniveau se résume en trois éléments (Courgeau, 2004). Ces trois aspects fondamentaux de la modélisation multiniveau sont la définition des groupes, la modélisation des effets fixes et la modélisation des effets aléatoires. 


\section{Présentation et discussion des résultats}

Les résultats montrent que sur un total de 811 cas retenus, 272 , soit $33 \%$, ont été victimes de l'abandon scolaire. 391 nont jamais été à l'école et seulement 148 chefs de ménages ont achevé au moins le cycle primaire. Au total, 48 \% de la population sont analphabètes au Sud-Kivu et l'on observe de différences importantes selon qu'on est en ville ou au village. Le nombre de ceux qui nont que le niveau primaire s'élève à 212, soit $26 \%$. Ceux ayant atteint le niveau d'études secondaire sont au nombre de 196, soit $24 \%$. Ceux qui ont un niveau d'études universitaire ne sont que 12, soit 1,5\%. En majorité (58\%), les gens ont arrêté les études pour cause des difficultés financières des parents. Il existe un lien étroit entre la pauvreté des ménages et le décrochage scolaire (Bouchard, 2001). Gueddari (2015) a trouvé que la situation de pauvreté dans le milieu rural au Maroc conduit les enfants à l'abandon scolaire précoce. À partir des années 1960, le budget alloué à l'enseignement au Congo n'a fait que chuter (Murhi Mihigo, 2016), passant de $7 \%$ à $1 \%$ du PIB et de $25 \%$ à moins de $5 \%$ du budget national, entraînant une diminution de $96 \%$ des dépenses par élève et par an dans les écoles primaires et secondaires (République démocratique du Congo, 2011).

Les résultats de l'analyse économétrique sont donnés dans le tableau ci-dessous.

\section{Tableau 5}

Résultats par niveau concernant la probabilité d'abandonner l'école au Sud-Kivu.

\begin{tabular}{|c|c|c|c|c|c|}
\hline Variables & $\begin{array}{c}\text { Modèle } 1 \\
\text { (niveau de l'élève) }\end{array}$ & $\begin{array}{c}\text { Modèle } 2 \\
\text { (niveau de la famille) }\end{array}$ & $\begin{array}{c}\text { Modèle } 3 \\
\text { (niveau de l'école) }\end{array}$ & $\begin{array}{c}\text { Modèle complet } \\
\text { avec les trois } \\
\text { niveaux }\end{array}$ & $\begin{array}{l}\text { Effets marginaux du } \\
\text { modèle global (dy/dx } \\
\qquad(P>|z|)\end{array}$ \\
\hline Constate & $-1,48(0,000)^{*}$ & $-0,696(0,012)^{* *}$ & $-1,330(0,751)$ & $-1,162(0,098)^{* * *}$ & \\
\hline AGELEVE & $0,008(0,920)$ & & & $0,004(0,640)$ & $0,0013(0,640)$ \\
\hline AGESCOL & $0,347(0,000)^{*}$ & & & $0,421(0,000)^{*}$ & $-0,1400(0,000)^{\wedge}$ \\
\hline ECHECSCOL & $1,53(0,000)^{*}$ & & & $1,215(0,000)^{*}$ & $0,284(0,000)^{\wedge}$ \\
\hline SEXE & $-0,28(0,000)^{*}$ & & & $-0,420(0,012)^{*}$ & $-0,150(0,017)^{\wedge}$ \\
\hline TMENAGE & & $0,020(0,225)$ & & $0,012(0,044)^{* *}$ & $0,0041(0,048)$ \\
\hline MILIEURES & & $0,90(0,000)^{*}$ & & $0,184(0,056)^{* * *}$ & $0,058(0,056)^{\wedge}$ \\
\hline PARENSEIG & & $0,009(0,046)^{* *}$ & & $0,001(0,055)^{* * *}$ & $0,0003(0,054)$ \\
\hline SITECONO & & $0,466(0,003)^{* *}$ & & $0,367(0,026)^{* *}$ & $0,132(0,025)^{\wedge}$ \\
\hline EDUCPARE & & $-0,233(0,015)^{* *}$ & & $-0,37(0,000)^{*}$ & $-0,124(0,000)^{\wedge}$ \\
\hline LANGUE & & $0,096(0,033)^{* *}$ & & $0,127(0,225)$ & $0,042(0,225)$ \\
\hline AGECHEFM & & $-0,018(0,000)^{*}$ & & $-0,021(0,000)^{*}$ & $-0,007(0,000)$ \\
\hline CONDUITENSEIG & & & $0,414(0,002)^{* *}$ & $0,332(0,029)^{* *}$ & $0,111(0,028)$ \\
\hline ABSENCENSEIGN & & & $0,089(0,060)^{* * *}$ & $0,001(0,994)$ & $0,0004(0,994)$ \\
\hline ACTIVPARASCOL & & & $0,055(0,669)$ & $0,091(0,520)$ & $0,030(0,519)^{\wedge}$ \\
\hline TRAITEMAENSEIG & & & $0,340(0,016)^{* *}$ & $0,259(0,009)^{*}$ & $0,086(0,0009)^{*}$ \\
\hline QUALIFIENSEIGN & & & $-0,458(0,000)^{*}$ & $-0,415(0,001)^{*}$ & $-0,138(0,000)^{*}$ \\
\hline REGIMECOL & & & $0,042(0,025)^{*}$ & $0,176(0,241)$ & $0,058(0,242)$ \\
\hline
\end{tabular}

${ }^{(*)}$ Significatif au seuil de $1 \%$. (**) Significatif au seuil de $5 \%$. $\left.{ }^{* * *}\right)$ Significatif à $10 \%$. ^ dy/dx est pour le changement discret de la variable dichotomique entre 0-1. 
En tout, dix-sept variables ont été intégrées dans l'analyse. Le modèle d'analyse multiniveau donne douze variables statistiquement significatives. Au niveau individuel, l'âge de l'élève, les échecs scolaires, le début de l'école avec retard influent positivement la probabilité d'abandonner précocement l'école au Sud-Kivu. Lorsqu'on a commencé les études à l'âge normal, la probabilité d'abandonner l'école avant la fin du cycle diminue de $14 \%$ alors que les échecs scolaires augmentent $28 \%$ la probabilité d'abandonner l'école. Ces résultats sont proches de ceux de Demba (2010) démontrant qu'au Gabon l'échec scolaire décourage les élèves et les conduit à la recherche d'alternatives. Le fait d'être une fille a un effet négatif sur la probabilité d'abandonner précocement l'école. La littérature montre que la probabilité d'abandonner des garçons est plus forte que celle des filles; nos résultats le confirment également. Ces résultats sont donc conformes à ceux d'Ananga (2011) et de Coulidiati-Kiélem (2009), qui montrent que le pourcentage des garçons qui abandonnent l'école est plus élevé que celui des filles. Les caractéristiques familiales ont pour la majorité une influence positive sur la décision de décrochage scolaire. L'éducation des parents est un élément important qui limite l'abandon scolaire des enfants. Les résultats montrent que lorsque les parents ont étudié, cela augmente la chance de $12 \%$ à l'élève de ne pas décrocher avant la fin du cycle. Moins les parents sont éduqués, plus leurs enfants peuvent abandonner. C'est ainsi que Belzil (2004) et Joanis (2002) montrent qu'il existe une relation positive entre l'éducation des parents et celle des enfants. La réalité d'abandon scolaire n'est donc pas loin d'être un problème intergénérationnel. La scolarité de la mère, en plus d'être associée aux aspirations scolaires, est reliée selon Durand (2006) à la performance des élèves. La variable taille du ménage a une influence positive sur la probabilité d'abandon scolaire. Pour Issidor (2006), le taux d'abandon est plus élevé dans les ménages de grande taille. Lorsqu'un élève vit dans une famille nombreuse, comme c'est le cas général au Congo où la moyenne est de six individus par ménage (République démocratique du Congo, 2011), il est plus exposé à abandonner l'école prématurément. Les mêmes résultats ont été trouvés par Boissonneault et al. (2007) au Canada. Le milieu de résidence est aussi positivement corrélé avec la probabilité d'abandon scolaire au Sud-Kivu. Un élève issu d'un milieu rural est plus soumis au décrochage précoce que celui vivant en ville. L'UNICEF montre que, sur 100 élèves inscrits en première année de l'enseignement primaire en milieu rural au Congo, 19 seulement arrivent en sixième année sans interrompre leurs études (UNICEF, 2011). L'UNESCO (2012) a trouvé aussi que 13 sur 100 élèves ayant commencé l'école primaire atteignent le premier cycle de l'école secondaire. Okumu et al. (2008) affirment qu'en Ouganda la probabilité d'abandonner l'école se réduit lorsqu'on passe de la région rurale vers la région urbaine. Nos résultats prouvent la même réalité. Au Congo, les écoles en milieu rural n'ont pas le minimum nécessaire en termes d'équipements et de matériels didactiques, de latrines, d'eau potable et d'électricité pouvant inciter les élèves à poursuivre leurs études. Le milieu rural est donc défavorisé par rapport au milieu urbain. La situation économique du ménage est aussi statistiquement significative et a une influence positive sur la probabilité d'abandon précoce de l'école. Puisque les dépenses de l'éducation ne sont pas couvertes totalement par l'État, les enfants issus des ménages économiquement faibles sont confrontés au problème de décrochage scolaire par manque de moyens. La langue maternelle a aussi une influence significative sur l'abandon scolaire. Les élèves qui viennent des familles où le français est la langue maternelle s'adaptent facilement et maîtrisent sans difficulté les rites scolaires que d'autres n'arrivent pas à maîtriser. 
Pour ce qui est des déterminants liés à l'environnement scolaire, la mauvaise conduite des enseignants joue positivement et significativement sur la probabilité d'abandon précoce de l'élève. Le rôle de l'enseignant est primordial dans l'éducation des enfants qui fréquentent l'école. Lorsqu'un enseignant cumule les absences, cela a une influence sur la décision d'abandonner pour un élève. La pratique au Congo veut que lorsqu'un titulaire d'une classe ne s'est pas présenté à l'école, les élèves suivent les cours dans une classe parallèle avec un autre enseignant. Ceci peut avoir comme conséquence, entre autres, l'inadaptation de certains élèves au nouveau titulaire. La qualification de l'enseignant limite jusqu'à $13 \%$ le décrochage scolaire, ce qui a été également constaté par l'UNESCO (2012). Un enseignant qualifié est plus professionnel que celui qui ne l'est pas. Le mauvais traitement de l'enseignant augmente la probabilité d'abandonner les études. Comme l'indique Issidor (2006), les recherches montrent que les enseignants non motivés peuvent être source d'abandons massifs des élèves ou alors peuvent conduire certains enseignants à quitter le secteur à la recherche d'un meilleur traitement. Un enseignant non motivé, comme le souligne Murhi Mihigo (2016), ne donne pas le meilleur de lui-même. Pour Jeffrey (2013), les rites scolaires renforcent les liens entre les enseignants et les élèves. Le climat dans lequel évolue un élève et les méthodes d'apprentissage peuvent jouer un rôle non négligeable dans la décision d'abandon scolaire. Enfin, le régime scolaire influence aussi la probabilité de décrocher. Dans la configuration actuelle de l'enseignement au Congo, les écoles non gérées directement par l'État (conventionnées et privées agréées) sont plus nombreuses que les écoles publiques (Murhi Mihigo, 2016). Dans une situation pareille, le régime de l'école peut conduire les élèves à abandonner faute de moyens pour financer les études dans les établissements privés sans subvention étatique. En somme, l'analyse multiniveau a été appliquée aux variables explicatives de l'étude : l'âge de l'élève, l'âge de l'élève au début de la scolarisation, l'échec scolaire, le sexe, la taille du ménage, le milieu de résidence, la part du revenu allouée à l'éducation des enfants, la situation économique du ménage, l'éducation des parents, la langue maternelle, l'âge du chef de ménage, la conduite de l'enseignant, l'absentéisme de l'enseignant, les activités parascolaires, le traitement des enseignants, leurs qualifications et le régime de gestion de l'école. Les résultats montrent que le niveau le plus prépondérant est celui de la famille (six sur sept variables), suivi de celui de l'école (cinq variables sur six) et enfin le niveau de l'élève (quatre variables sur cinq). Le contexte scolaire est donc un élément important dans l'analyse de l'abandon scolaire. Les études traitant la question de l'abandon scolaire se focalisent rarement sur l'environnement scolaire.

\section{Conclusion}

La littérature montre clairement que le décrochage scolaire est un phénomène international et multidisciplinaire. Chaque discipline lui consacre des études et des enquêtes centrées sur un angle particulier. Le présent article avait pour but de décrire l'ampleur, les caractéristiques ainsi que les facteurs explicatifs du décrochage scolaire au Sud-Kivu en République démocratique du Congo. Les données de la division provinciale de l'EPSP montrent que le taux d'admission a largement augmenté depuis 2005 en première année d'enseignement primaire. Les effectifs sont passés de 316223 garçons en 2005 à 420868 garçons en 2009; et de 291419 filles en 2005 à 370590 filles en 2009. Le taux de scolarisation à l'école secondaire est plus faible que celui de l'école primaire. Les données de l'enquête 1-2-3 nous ont permis de constituer, en les combinant aux données de l'EPSS, un échantillon de 811 observations. Après une analyse économétrique et nous appuyant sur un modèle 
d'analyse multiniveau, avec trois niveaux d'analyse, nous avons trouvé que douze sur les dix-sept variables retenues étaient statistiquement significatives dans l'explication du phénomène étudié dans le modèle complet. Les caractéristiques individuelles, familiales et scolaires ont une forte influence sur la probabilité d'abandon scolaire d'un élève avant la fin du cursus entamé au Sud-Kivu. La perspective de cette recherche est double. Premièrement, il faudrait chercher à intégrer l'effet variable, au cours du temps, des facteurs d'abandon dans un modèle de durée semi-paramétrique. Deuxièmement, l'utilisation et la construction d'autres enquêtes spécialement construites pour l'étude des parcours scolaires permettraient d'élargir les facteurs influençant le décrochage et ainsi s'intéresser aussi au décrochage en milieu universitaire. Pour ce faire, la constitution des cohortes est importante pour un suivi non biaisé et intégrant toutes les variables.

\section{Notes}

1 Nous avons présenté cette communication au colloque organisé par le Laboratoire d'économie appliquée au développement (LEAD) de l'Université catholique de Bukavu en collaboration avec le PNUD en juin 2015.

2 Selon Boutin (2010) et Coulidiati-Kiélem (2009), les modèles multiniveaux sont conçus à l'origine pour modéliser des données qui ont une structure hiérarchisée, c'est-à-dire qui sont constituées de niveaux emboîtés les uns dans les autres, où les micro-unités n'appartiennent qu'à une seule unité de niveau supérieur.

3 Les décrocheurs involontaires sont ceux qui ont tout le potentiel intellectuel pour terminer leurs études, mais où l'environnement dans lequel ils évoluent les contraint à abandonner.

4 Voir l'annuaire de la Division provinciale de l'enseignement primaire, secondaire et professionnel (la dénomination a changé depuis 2014 et est devenue " enseignement primaire, secondaire et initiation à la nouvelle citoyenneté »).

5 Le taux d'admission va de pair avec la capacité d'accueil des élèves dans une classe et dans un établissement...

6 Le taux d'admission va de pair avec la capacité d'accueil des élèves dans une classe et dans un établissement...

$7 \quad$ Il sied de signaler que les chiffres concernant la population scolarisable sont issus d'une estimation (le dernier recensement général de la population en République démocratique du Congo remonte aux années 1980).

8 Nous ne disposons pas des taux de scolarisation des filles et garçons par territoire, ce qui nous permettrait de comparer le milieu rural et urbain.

9 La constitution d'une base des données dans le domaine de l'éducation est plus qu'une urgence. Il faut pouvoir faire le suivi par exemple des cohortes sur une durée relativement longue. Pour ce faire, nous allons communiquer avec les différents partenaires dans ce secteur.

10 Nous devons souligner que le nombre de filles qui sont dans le système scolaire reste très bas en dépit de la multiplication des campagnes qui appellent à la mobilisation de tous pour garantir une égalité des sexes.

11 Le modèle Probit univarié mesure de la probabilité qu'un événement se réalise. Les deux auteurs ont aussi utilisé dans leurs travaux ce modèle. Plus précisément, ce modèle est développé dans le cours d'économétrie approfondie de Beine (2009).

12 Les variables explicatives sont inspirées de la littérature et du contexte. Seules les variables présentes dans les bases des données ont été intégrées dans l'analyse. 


\section{Références}

Ananga, E. D. (2011). Typology of school dropout: The dimensions and dynamics of dropout in Ghana. International Journal of Educational Development, 31(4), 374-381. http://dx.doi.org/10.1016/j.ijedudev.2011.01.006

Belzil, C. (2004). Un modèle économétrique dynamique de l'abandon scolaire au Québec et en Ontario. L'Actualité économique, 80, (2-3), 363-381. http://dx.doi.org/10.7202/011391ar

Benny, M. et Frappier, J.-Y. (1997). L'abandon scolaire. Extraits du PRO-ADO (vol. 6). ROCIDEC.

Boissonneault, J., Michaud, J., Côté, D., Tremblay, C.-L. et Allaire, G. (2007). L'abandon scolaire en Ontario français et perspectives d'avenir des jeunes. Éducation et francophonie, 35(1), 3-22. Repéré à http://www.acelf.ca/c/revue/pdf/XXXV 1 003.pdf

Bouchard, I. (2001). Les milieux à risque d'abandon scolaire: Quand pauvreté, conditions de vie et décrochage scolaire vont de pair. Repéré à http://www.crepas.qc.ca/userfiles/ancien site/editeur10/DOC 9 16.pdf

Bourbonnais, R. (2009). Économétrie : Manuel et exercices corrigés. Paris : Dunod.

Boutin, D. (2010). La transition des jeunes camerounais vers le marché du travail. Repéré à http://ged.u-bordeaux4.fr/ceddt152.pdf

Bressoux, P., Coustère, P. et Leroy-Audouin, C. (1997). Les modèles multiniveau dans l'analyse écologique : le cas de la recherche en éducation. Revue française de sociologie, 38(1), 67-96. http://dx.doi.org/10.2307/3322373

Bushnik, T., Barr-Telford, L. et Bussière, P. (2004). In and out of high school: First results from the second cycle of the Youth in Transition Survey. Repéré à http://www.statcan.gc.ca/pub/81-595-m/81-595-m2004014-eng.pdf

Chávez, R. C., Belkin, L. D., Hornback, J. G. et Adams, K. (1991). Dropping out of school: issues affecting culturally, ethnically, and linguistically distinct student groups. The Journal of Educational Issues of Language Minority Students, 8 , 9-21. Repéré à http://ci.education.nmsu.edu/files/2014/01/1_DroppingOutOfSchool.pdf

Coulidiati-Kiélem, J. (2009). Les effets du contexte scolaire sur les performances des élèves au collège au Burkina Faso: analyse multiniveau. Repéré à http://ged.u-bordeaux4.fr/ceddt149.pdf

Courgeau, D. (2004). Du groupe à l'individu : synthèse multiniveau. Paris : Institut national d'études démographiques.

Dagenais, M., Montmarquette, C., Viennot-Briot, N. et Meunier, M. (2000). Le décrochage scolaire, la performance scolaire et le travail pendant les études: un modèle avec groupe hétérogène. Repéré à http://www.cirano.qc.ca/pdf/publication/2000s-55.pdf

Dei, G. J. S., Mazzuca, J., Mclsaac, E. et Zine, J. (1997). Reconstructing « dropout »: A critical ethnography of the dynamics of Black students disengagement from school. Toronto, $\mathrm{ON}$ : University of Toronto Press.

Demba, J. J. (2010). La face subjective de l'échec scolaire : récits d'élèves gabonais du secondaire (Thèse de doctorat, Université Laval, Québec). Repéré à http://theses.ulaval.ca/archimede/fichiers/27445/27445.pdf

Diagne, A., Kafando, I. et Ounteni, M. (2006). Pourquoi les enfants quittent-ils l'école? Un modèle hiérarchique multinomial des abandons dans l'éducation primaire au Sénégal. Les Cabiers du SISERA, (8). Repéré à http://www.cres-sn.org/sites/default/files/pourquoi les enfants quittent-ils.pdf

Duchesne, K. et Thomas, D. (2005). Le décrochage scolaire dans la Commission scolaire de Rouyn-Noranda. Repéré à http://www.uqat.ca/telechargements/info entites/Rapport\%20de\%20diffusion\%20-\%20Projet\%20Raccrochage.pdf

Durand, M.-H. (2006). Les enfants non scolarisés en milieu urbain : une comparaison des déterminants intra familiaux, inter familiaux et des effets de voisinage dans sept capitales ouest africaines. Repéré à https://en.ird.fr/content/view/full/50788

Easterly, W. (2008). Can the West save Africa?. http://dx.doi.org/10.3386/w14363

Elliot, D. S. et Voss, H. L. (1974). Delinquency and dropout. Lexington, MA : Lexington Books.

Forget, A. et Lehraus, K. (2015). La différenciation en classe : qu'en est-il des pratiques réelles des enseignants? Formation et profession, 23(3), 70-84. http://dx.doi.org/10.18162/fp.2015.287 
Fry, R. (2003). Hispanic youth dropping out of U.S. schools: Measuring the challenge. Repéré à http://www.pewhispanic.org/files/reports/19.pdf

Gueddari, K. (2015). L'abandon scolaire en milieu rural marocain : une analyse interactionniste du point de vue des familles (Mémoire de maîtrise, Université de Montréal). Repéré à https://papyrus.bib.umontreal.ca/xmlui/handle/1866/13792

Huot, A. et Castonguay, C. (2014). Processus et résultats sommaires d'une intervention collaborative dans le passage primaire-secondaire en milieu défavorisé rural. Formation et profession, 23(1), 11-23. http://dx.doi.org/10.18162/fp.2015.123

Isambert-Jamati, V. (1992). Quelques rappels de l'émergence de l'échec scolaire comme « problème social ». Dans B. Pierrehumbert (dir.), L'échec à l'école, échec de l'école?. Paris.

Issidor, N. (2006). Un profil de l'abandon scolaire au Cameroun. Repéré à http://en.dial.ird.fr/content/download/88141/672642/version/1/file/22 issidornoumba.pdf

Jeffrey, D. (2013). Rites scolaires et identité d’élève. Formation et profession, 21(1), 50-64 http://dx.doi.org/10.18162/fp.2013.26

Jimerson, S., Egeland, B., Sroufe, L. A. et Carlson, B. (2000). A prospective, longitudinal study of high school dropouts: Examining multiple predictors across development. Journal of School Psychology, 38(6), 525-549. http://dx.doi.org/10.1016/s0022-4405(00)00051-0

Joanis, M. (2002). L'économie de l'éducation : méthodologies, constats et leçons. Repéré à http://www.cirano.qc.ca/pdf/publication/2002s-70.pdf

Larivée, S. J. et Garnier, P. (2014). Les relations école-famille et la formation des enseignants du primaire : éléments de comparaison France - Québec. Formation et profession, 22(1), 27-40. http://dx.doi.org/10.18162/fp.2014.31

Lee, V. E. et Burkam, D. T. (2000, décembre). Dropping out of high school: The role of school organization and structure. Communication présentée à la conférence « Dropouts in America: How severe is the problem? What do we know about intervention and prevention? ", Cambridge, MA. Repéré à https://www.civilrightsproject.ucla.edu/research/ k-12-education/school-dropouts/dropping-out-of-high-school-the-role-of-school-organization-and-structure/leerole-school-organization-2001.pdf

Lloyd, C. B., Mensch, B. S. et Clark, W. H. (2000). The effects of primary school quality on school dropout among Kenyan girls and boys. Comparative Education Review, 44(2), 113-147. http://dx.doi.org/10.1086/447600

Marcotte, D. E. (2013). High school dropout and teen childbearing. Economics of Education Revierw, 34, $258-268$. http://dx.doi.org/10.1016/j.econedurev.2013.01.002

Murhi Mihigo, I. (2016). Privatisation du secteur éducatif en République démocratique du Congo : causes et conséquences. Dans J. Bobineau et P. Gieg (dir.), The Democratic Republic of the Congo : Problems, progress and prospects (volume 55). Berlin : LIT.

Musisi, N. B., Kasente, D. et Balihuta, A. M. (2008). Attendance patterns and causes of dropout in primary schools in Uganda: a case study of 16 schools, a research report. Kampala : Makerere University Research Repository.

Muskens, G. (2009). Inclusion and education in European countries. Repéré à http://www.kcco.nl/doc/kennisbank/inclusion education_EU comparative.pdf

Ntagoma, J. P. et Lukuli, J.-C. (2008). Les abandons scolaires au Sud-Kivu : causes, motivations à l'inscription et mécanismes alternatifs de lutte.

Okumu, I. M., Nakajjo, A. et Isoke, D. (2008). Socioeconomic determinants of primary school dropout: the logistic model analysis. Repéré à https://mpra.ub.uni-muenchen.de/7851/1/MPRA paper 7851.pdf

République démocratique du Congo. (2011). Rapport du cadrage macroéconomique et budgétaire. Kinshasa : Ministère du Plan, Document de la stratégie de croissance et de réduction de la pauvreté (DSCRP).

Rosenthal, B. S. (1998). Non-school correlates of dropout: An integrative review of the literature. Children and Youth Services Review, 20(5), 413-433. http://dx.doi.org/10.1016/s0190-7409(98)00015-2 
Rumberger, R. (1995). Dropping out of middle school: A multilevel analysis of students and schools. American Educational Research Journal, 32(3), 583. http://dx.doi.org/10.2307/1163325

Sabates, R., Akyeampong, K., Westbrook, J. et Hunt, F. (2010). School dropout: Patterns, causes, changes and policies. Repéré à http://unesdoc.unesco.org/images/0019/001907/190771e.pdf

Sabates, R., Hossain, A. et Lewin, K. M. (2010). School dropout in Bangladesh: New insights from longitudinal evidence. Repéré à http://www.create-rpc.org/pdf documents/PTA49.pdf

Sawadogo, J. B. et Soura, A. B. (2002). L'abandon précoce en milieu scolaire : Analyse et recherche de modèle explicatif. Repéré à http://www.rocare.org/smallgrant burkina2002.pdf

Sum, A., Khatiwada, I., McLaughlin, J. et Palma, S. (2009). The consequences of dropping out of high school: Joblessness and jailing for high school dropouts and the high cost for taxpayers. Repéré à https://repository.library.northeastern.edu/downloads/neu:376324?datastream id=content

UNESCO. (2009). Forum mondial sur l'éducation. Paris.

UNESCO. (2010). Services sociaux de base et capital humain en Afrique subsabarienne. Paris.

UNESCO. (2012). Opportunités perdues : Impact du redoublement et du départ prématuré de l'école. Repéré à http://uis.unesco. $\mathrm{org} / \mathrm{sites} /$ default/files/documents/opportunities-lost-the-impact-of-grade-repetition-and-early-school-leaving-fr.pdf

UNICEF. (2011). Objectifs du Millénaire pour le développement : Rapport de 2011. Repéré à http://www.un.org/fr/millenniumgoals/pdf/report 2011.pdf

Vitaro, F., Larocque, D., Janosz, M. et Tremblay, R. E. (2001). Negative social experiences and dropping out of school. Educational Psychology, 21(4), 401-415. http://dx.doi.org/10.1080/01443410120090795

\section{Pour citer cet article}

Murhi Mihigo, I. et Bucekuderhwa Bashige, C. (2017). Abandon scolaire au Sud-Kivu. Formation et profession, 25(2), 49-64. http://dx.doi.org/10318162/fp.2017.418 